\section{Innovación docente: catorce años de experiencia en un curso teórico de pregrado en medicina}

\author{
PAULINE BÖHM ${ }^{1}$, ANDRÉS SOFFIA ${ }^{1}$, LUIS ANTONIO DÍAZ ${ }^{2}$, \\ IGNACIO VILLAGRÁN ${ }^{3}$, MARGARITA PIZARRO ${ }^{2}$, \\ LUZ MARÍA COLLINS ${ }^{4}$, ARNOLDO RIQUELME ${ }^{2,3,4}$, HUGO MONRROY ${ }^{2}$
}

\section{Teaching innovation in an undergraduate theoretical medicine course}

Background: The School of Medicine of the Pontificia Universidad Católica de Chile implemented diverse curricular changes addressing teaching challenges, including those related to generational diversity. Aim: To describe the implementation and results of curricular innovation in the Theoretic Gastroenterology Course (CTG) imparted between 2008 and 2020. Materials and Methods: The new teaching methods consisted in the implementation of interactive sessions, research conferences, video-recorded classes, and a learning management/assessment platform. An assessment of the learning model was implemented. As bibliographic material we incorporated self-instructive material and the CTG manual was re-edited. We registered the course syllabi, evaluation surveys, and final grades. Results: Students dedicated more time to attend the course, from 12.2 hours before to 18 hours after the implementation of video lessons $(p<0.05)$. They reported improvements in the areas "Feedback" (from 6.2 to 6.6, on a scale of 1 to $7 ; p<0.05$ ) and "Grades" (from 6.3 to $6.4 ; p<0.05$ ), after implementing a learning model assessment. The score for "Information sources" increased from 6.5 to 6.6 after the re-edition of the manual $(p<0.05)$. The final grades were similar or significantly higher than the average grades of all the theoretical courses imparted in the same period. Conclusions: The CTG underwent a series of curricular modifications, allowing for a rapid adaptation to extremely dynamic academic conditions.

(Rev Med Chile 2020; 148: 1659-1667)

Key words: Curriculum; Educational Measurement; Education, Premedical; Students; Teaching.
${ }^{1}$ Escuela de Medicina, Pontificia Universidad Católica de Chile.

Santiago, Chile.

${ }^{2}$ Departamento de

Gastroenterología, Escuela de Medicina, Pontificia Universidad Católica de Chile. Santiago, Chile. ${ }^{3}$ Departamento de Ciencias de la Salud, Pontificia Universidad Católica de Chile. Santiago, Chile. ${ }^{4}$ Centro de Educación Médica y Ciencias de la Salud, Pontificia Universidad Católica de Chile. Santiago, Chile.

Trabajo no recibió financiamiento. Los autores declaran no tener conflictos de interés.

Recibido el 25 de mayo de 2020, aceptado el 13 de octubre de 2020

Correspondencia a:

Dr. Hugo Monrroy Departamento de Gastroenterología, Pontificia Universidad Católica de Chile. Diagonal Paraguay 362, piso 4. Santiago, Chile. hmonrroy@med.puc.cl
L a Escuela de Medicina de la Pontificia Universidad Católica de Chile (EMPUC) ha llevado a cabo diversos cambios curriculares para dar respuesta a los desafíos que surgen en docencia en Medicina. Como parte del desarrollo de evaluación continua del proceso enseñanza-aprendizaje, el año 2015 implementó una reforma curricular innovadora que establece un nuevo perfil del egresado y se orienta por metas de aprendizaje, entre otras características (Tabla 1). Además, existe una evaluación y armonización permanente de los contenidos y actividades curriculares, a fin de promover una formación y desarrollo integral. Como parte de esta reforma, la carrera fue acortada de una duración tradicional de siete a seis años ${ }^{1}$.

Resulta necesario que los programas de pregrado sepan hacerse cargo de la diversidad de estilos de aprendizaje del estudiantado, considerando que en los estudiantes de medicina inicialmente predomina un estilo de aprendizaje abstracto-reflexivo, que evoluciona con los años a uno abstracto-activo ${ }^{2,3}$. 
Tabla 1. Características principales de la reforma curricular del año 2015 de la carrera de Medicina, Pontificia Universidad Católica de Chile

- Enfoque centrado en competencias

- Integración horizontal y vertical de los contenidos y competencias

- Gran énfasis en aprendizaje y evaluación continua de profesionalismo médico y competencias transversales, tales como la comunicación

- Metodologías docentes activas, centradas en el estudiante, con énfasis en simulación, aprendizaje por pares, flipped classroom, etc

- Evaluaciones ajustadas a los contenidos y las metodologías

- Énfasis en el bienestar estudiantil y de la comunidad

La diversidad generacional constituye un desafío a la hora de formular un diseño curricular. Los millennials, nacidos entre 1980 y 2000, que corresponden a la generación cursando actualmente estudios de medicina, son frecuentemente criticados por su impaciencia, distractibilidad, sobre-involucramiento social, entre otras características. Sin embargo, destacan por un profundo empoderamiento, colaboratividad, innovación, y mayor percepción de satisfacción frente a la obtención de resultados e implementación de estos $^{4}$. La educación de pregrado debe reconocer y valorar este cambio generacional como un desafío, innovando en la generación de instancias de aprendizaje productivas.

En el diseño curricular tradicional (vigente hasta 2014), el capítulo teórico de Gastroenterología (CTG) se enmarca dentro de las actividades del curso Integrado de Clínica III y IV, impartiéndose durante el primer semestre de cuarto año de carrera. Por otro lado, en el currículo reformado (vigente a partir de 2015) es incluido dentro del curso Clínicas II y III, dictándose durante el segundo semestre del tercer año o el primer semestre del cuarto año de la carrera. El capítulo tiene carácter obligatorio, siendo parte de los requisitos mínimos para aprobar el año académico.

Históricamente, el CTG ha sido sometido a cambios curriculares, destacando la generación de apuntes para el estudiante ("Temas de Gastroenterología Médica”) en 2005 y la mejora sistemática de las evaluaciones entre los años 2002 y 2006, logrando un $100 \%$ de cobertura de los temas (validez de contenido), ausencia de violaciones de construcción de pruebas de selección múltiple y confiabilidad de 0,78 (Alfa de Cronbach) ${ }^{5}$. Además, el año 2007 se incorporó el Portafolio, una colección de trabajos que permite la aplicación del conocimiento y habilidades adquiridas sobre patología gastrointestinal, acompañado de un proceso de reflexión y pensamiento crítico, tributando al perfil de egreso de la EMPUC $^{6,7}$.

El objetivo de este artículo es reseñar la experiencia de innovación curricular que ha entregado el CTG a lo largo del período comprendido entre los años 2008-2020, de acuerdo con la percepción de los estudiantes y el impacto académico que han tenido los distintos cambios curriculares.

\section{Materiales y Métodos}

Se utilizó como fuente de información los registros del CTG entre 2008 y 2020, incluyendo los programas de cada capítulo, las encuestas de evaluación del capítulo en base a la percepción de los alumnos y las calificaciones de los alumnos. Los datos las encuestas de los estudiantes fueron completamente anonimizados. La información se obtuvo desde el Centro de Educación Médica (CEM) y la Dirección de Pregrado de la EMPUC.

\section{Programa del capitulo}

Se revisaron los programas de cada año del período estudiado. Se evaluaron dirigidamente las fechas y horarios destinados para clases lectivas y videograbadas, clases de integración, seminarios, evaluaciones y feedback, entre otras actividades docentes. Las clases lectivas fueron de asistencia opcional, solo fue obligatoria la participación en seminarios de casos clínicos. Se construyeron mapas conceptuales con la información contenida en los programas, incluyendo una línea del tiempo con los cambios realizados al programa (Figura 1), las actividades descritas en el último programa (Figura 2) y su distribución porcentual (Figura 3).

\section{Encuesta de evaluación del capítulo en base a la percepción de los alumnos}

Se aplicó una encuesta al final de cada capítulo, que se utiliza en todos los cursos teóricos y prácticos de la EMPUC, posterior a la evaluación final ${ }^{8}$. 
Se accedió al informe de cada capítulo realizado por el CEM, donde se resume el promedio de las respuestas de los alumnos de un mismo período en un único documento (datos por curso anonimizados, sin respuestas individuales).

\section{Calificaciones finales de los alumnos}

Se obtuvo como universo los registros de calificaciones individuales de todos los alumnos que han cursado el CTG en el período descrito y se calcularon los promedios de cada capítulo por

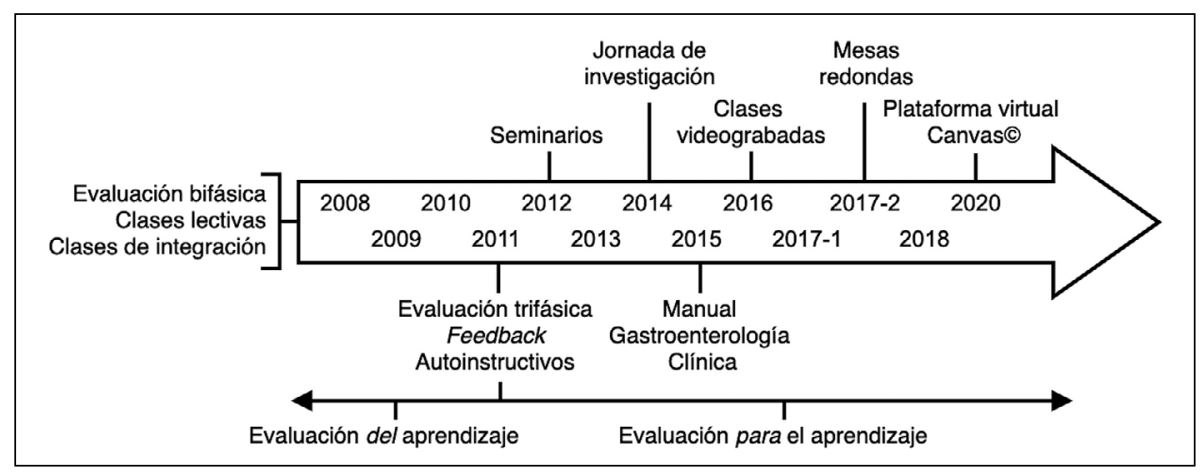

Figura 1. Línea del tiempo de los cambios realizados al programa del CTG entre los años 2008 y 2020.
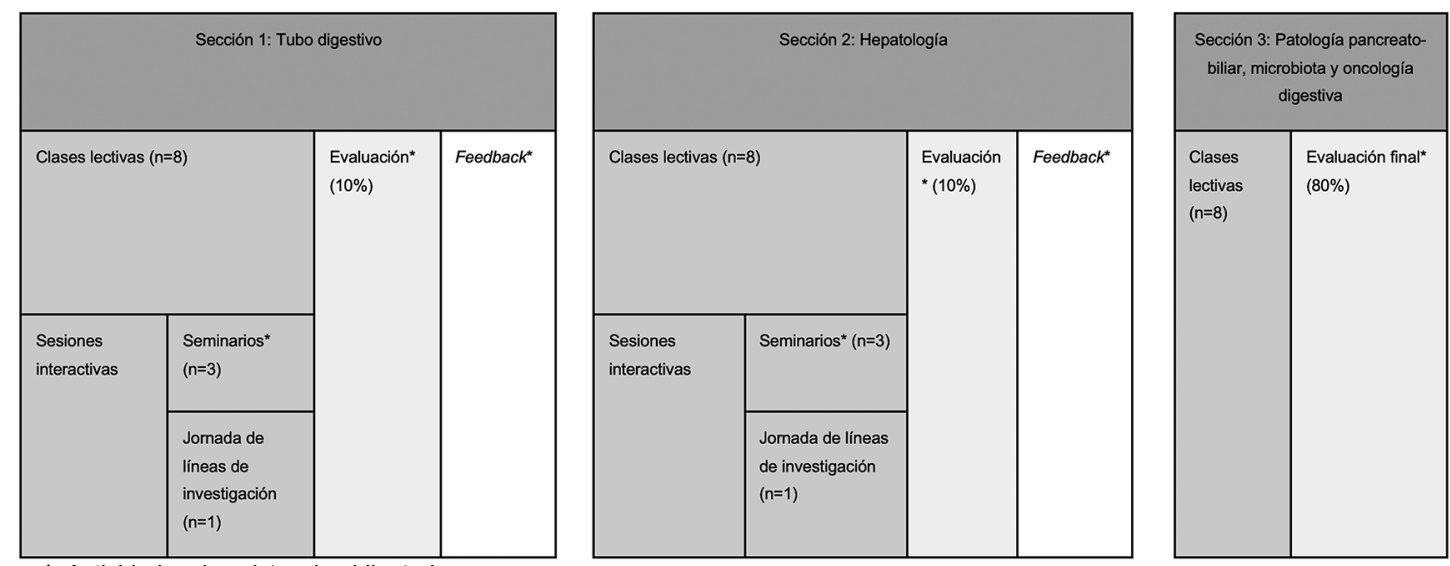

Figura 2. Distribución de actividades en el último programa del CTG (2020).

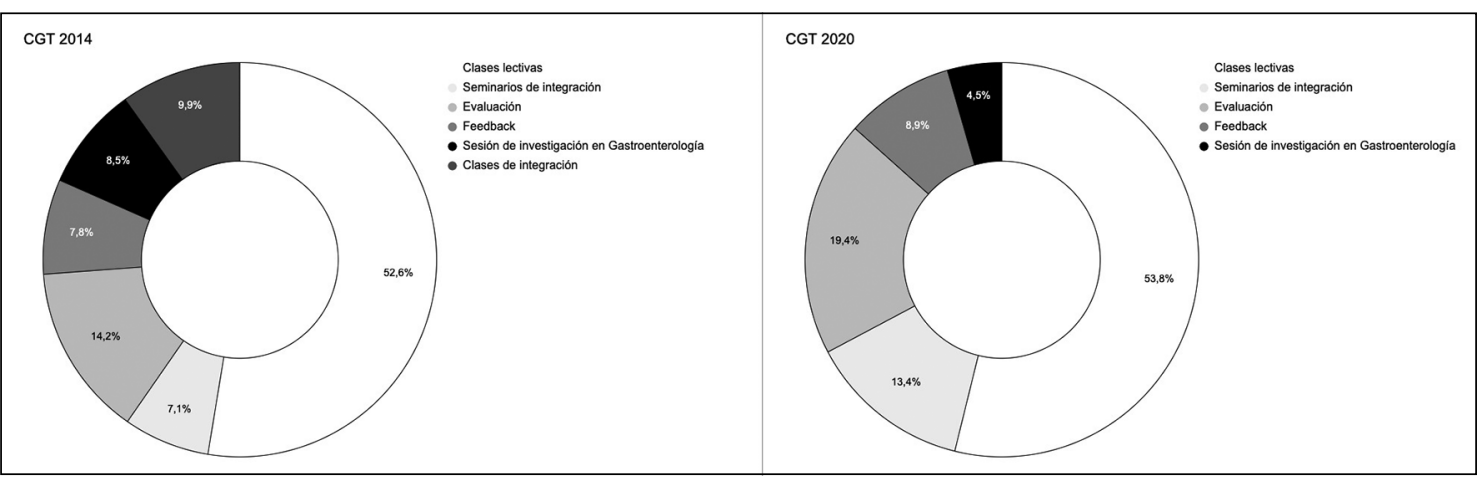

Figura 3. Distribución porcentual de actividades del programa del CTG pre y post reforma curricular 2015. Años 2014 vs 2020. 
separado. Para ponderar posibles efectos confundentes, se rescataron los promedios globales del componente teórico del curso Integrado de Clínica III y IV / Clínicas II y III durante el mismo período de tiempo y se compararon con los promedios del CTG.

\section{Análisis estadístico y uso de los datos}

Se reportaron las variables continuas mediante el promedio \pm desviación estándar. Las variables cualitativas fueron comparadas utilizando test $\mathrm{t}$ de student para muestras independientes. El análisis fue realizado de manera anónima por 2 investigadores que no están involucrados en la evaluación de los alumnos de pregrado. Se utilizó el software SPSS versión 24.0 (Armonk, NY: IBM Corp.). Este estudio fue aprobado por el Comité Ético Científico de la Pontificia Universidad Católica de Chile.

\section{Resultados}

\section{Programa}

Se analizaron los 13 programas del capítulo correspondientes al período 2008-2020. En ellos se observó que el horario del CTG contempla en promedio cuatro tardes a la semana, de 14:00 a 17:00, durante un período de dos a tres semanas (10-15 días hábiles). En la Tabla 2 se desglosan los cambios identificados en el programa del capítulo a lo largo del tiempo, mientras que en la Figura 1 se expone la temporalidad de su implementación.

Actualmente, el CTG consta de tres secciones (Tubo digestivo, Hepatología e Integración), cada una de las cuales cuenta con diversas actividades que se describen en la Figura 2. En la Figura 3 se detalla la distribución porcentual del tiempo destinado a cada una de ellas.

\section{Métodos docentes}

- Desarrollo de sesiones interactivas en base a casos clínicos: en el programa actual se incluyen seminarios de pequeño grupo $(n=3$, desde 2012), aunque en versiones previas del capítulo se realizaron también clases de integración y mesas redondas.

- Creación de la sección de investigación en Gastroenterología: a partir del año 2014 se incorpora a las actividades obligatorias del capítulo una jornada de investigación, en la que durante una tarde completa se exponen líneas de trabajo y proyectos por parte de docentes del departamento. En 2020 esta actividad se reestructuró a dos módulos electivos (jornadas de líneas de investigación) que se llevan a cabo en las secciones de Tubo digestivo y Hepatología.

- Incorporación de clases videograbadas: desde el año 2016, el capítulo se complementa con clases videograbadas expositivas que permiten el abordaje de distintos tópicos. A través de una plataforma virtual, se ponen a disposición del estudiantado 36 sesiones con una duración total de 17:18 horas, distribuidas en videos de Digestivo alto y bajo $(\mathrm{n}=16)$, Hepatología $(\mathrm{n}=15)$ y Cánceres en Gastroenterología $(\mathrm{n}=5)$, junto con 5 videos complementarios. Los alumnos pueden descargar las presentaciones y grabaciones de voz, correspondientes a cada sesión.

- Implementación de nueva plataforma de gestión de aprendizaje $\left(\right.$ Canvas $\left.^{\oplus}\right)$ : El año 2019 se iniciaron las capacitaciones a docentes para el uso de una nueva plataforma virtual, con el fin de explotar su potencial para el proceso de enseñanza y aprendizaje. La plataforma se implementó oficialmente desde el año 2020.

Tabla 2. Intervenciones realizadas al programa del Capítulo Teórico de Gastroenterología (CTG) entre los años 2008-2020

\begin{tabular}{|ll}
\hline Métodos docentes & $\begin{array}{l}\text { Desarrollo de sesiones interactivas en base a casos clínicos } \\
\text { Creación de sección de investigación en Gastroenterología } \\
\text { Incorporación de clases videograbadas } \\
\text { Implementación de nueva plataforma de gestión de aprendizaje }\left(\text { Canvas }^{\odot}\right)\end{array}$ \\
\hline Evaluación & Evaluación para el aprendizaje \\
Material bibliográfico & Uso de autoinstructivos \\
& Reedición del Manual de Gastroenterología Clínica
\end{tabular}




\section{Evaluación}

Desde el 2011 se inició una transición desde un enfoque de evaluación del aprendizaje a uno para el aprendizaje ("evaluación para el aprendizaje"). ${ }^{8}$ La intervención contempló el reemplazo de las evaluaciones sumativas de "Tubo digestivo" y "Hepatología" (50\% de la nota final cada una) por evaluaciones intermedias $(10 \%$ de la nota final cada una). Las evaluaciones sumativas son seguidas por sesiones de feedback dirigidas por el profesor a cargo del capítulo, en las que el alumnado puede aclarar dudas respecto a las preguntas de las pruebas $\mathrm{u}$ otras interrogantes que hayan surgido a lo largo del capítulo. Finalmente, se agrega una tercera evaluación integrativa, con una ponderación de $80 \%$ de la nota final.

\section{Material bibliográfico}

- Uso de autoinstructivos: a partir del año 2011 se pone a disposición del estudiantado presentaciones virtuales con preguntas formativas sobre temas tratados en clases.

- Reedición del Manual de Gastroenterología Clínica: desde el año 2005 se cuenta con "Apuntes de Temas de Gastroenterología Médica" como material complementario al capítulo. En 2015 estos se reeditaron y publicaron en forma de un Manual de Gastroenterología. Los capítulos de revisión obligatoria se encuentran explicitados en el programa del CTG.
Encuestas de evaluación del capítulo en base a la percepción de los alumnos

Este instrumento está compuesto por tres dominios: 1) Tiempo dedicado al capítulo fuera del horario establecido en el programa ( $\mathrm{h} /$ semana); 2) Logro de objetivos (\%) y 3 . Siete sub-dominios evaluados independientemente: Métodos, Profesores, Fuentes de Información, Feedback, Notas, Organización, Infraestructura y Materiales (escala Likert de 1 a 7). El promedio de estos subdominios se denomina "nota promedio". Adicionalmente, el alumno otorga una "nota global" al capítulo en la misma escala y dispone del reverso de la encuesta para comentarios libres.

En el dominio "Tiempo", los alumnos reportaron un mayor tiempo de dedicación al capítulo tras la implementación de las clases videograbadas, a pesar de una similar duración en semanas Este ítem se refiere al tiempo global utilizado, que incluye el tiempo utilizado en clases presenciales, y al estudio independiente, considerando el tiempo utilizado en clases videograbadas y lectura complementaria (Tabla 3). Al comparar los períodos pre y post implementación del paradigma de evaluación para el aprendizaje, se observó una mejora en las evaluaciones asignadas a los subdominios de "Feedback" y "Notas" (Tabla 4). Finalmente, tras reeditar e incorporar el Manual de Gastroenterología Clínica como parte de la bibliografía obligatoria del capítulo, se observó un alza en la evaluación del

Tabla 3. Autorreporte del tiempo (h/semana) destinado por los alumnos (n) al Capítulo Teórico de Gastroenterología (CTG) antes (2008-2015) y después (2016-2020) de la implementación de clases videograbadas

\begin{tabular}{|c|c|c|c|c|c|}
\hline \multirow[t]{2}{*}{ Parámetro } & \multicolumn{2}{|c|}{$\begin{array}{c}\text { Pre clases videograbadas } \\
(2008-2015)\end{array}$} & \multicolumn{2}{|c|}{$\begin{array}{c}\text { Post clases videograbadas } \\
(2016-2020)\end{array}$} & \multirow[t]{2}{*}{ Valor $p$} \\
\hline & $\mathbf{n}$ & Media \pm DE & $\mathbf{n}$ & Media \pm DE & \\
\hline Tiempo (h/semana) & 875 & $12,18 \pm 3,02$ & 513 & $17,5 \pm 4,81$ & $<0,05$ \\
\hline
\end{tabular}

Tabla 4. Calificación otorgada por los alumnos del Capítulo Teórico de Gastroenterología (CTG) en los subdominios de la encuesta de evaluación final del capítulo "Feedback" y "Notas" antes (2008-2010) y después (2011-2020) de la implementación del sistema de evaluación para el aprendizaje

\begin{tabular}{|lccccc|}
\hline Parámetro & $\begin{array}{c}\text { Evaluación del aprendizaje } \\
\text { (2008-2010) }\end{array}$ & $\begin{array}{c}\text { Evaluación para el aprendizaje } \\
\text { (2011-2020) }\end{array}$ & Valor $\mathbf{p}$ \\
Feedback & $\mathbf{n}$ & Media \pm DE & n & Media \pm DE & \\
Notas & 303 & $6,23 \pm 0,73$ & 1.085 & $6,64 \pm 0,24$ & $<0,05$ \\
\hline
\end{tabular}


Tabla 5. Calificación otorgada por los alumnos del Capítulo Teórico de Gastroenterología (CTG) en el subdominio de la encuesta de evaluación final del capítulo "Fuentes de información" antes (2008-2014) y después (2015-2020) de la publicación del Manual de Gastroenterología Clínica del capítulo

\begin{tabular}{|c|c|c|c|c|c|}
\hline \multirow[t]{2}{*}{ Parámetro } & \multicolumn{2}{|c|}{$\begin{array}{l}\text { Pre Manual de Gastroentero- } \\
\text { logía Clínica (2008-2014) }\end{array}$} & \multicolumn{2}{|c|}{$\begin{array}{l}\text { Post Manual de Gastroentero- } \\
\text { logía Clínica }(2015-2020)\end{array}$} & \multirow[t]{2}{*}{ Valor $p$} \\
\hline & $\mathbf{n}$ & Media $\pm \mathrm{DE}$ & n & Media \pm DE & \\
\hline Fuentes de información & 757 & $6,45 \pm 0,2$ & 631 & $6,63 \pm 0,22$ & $<0,05$ \\
\hline
\end{tabular}

Tabla 6. Promedio de calificaciones obtenidas en el Capítulo Teórico de Gastroenterología (CTG) y en el curso teórico (Integrado de Clínica III y IV / Clínicas II y III) entre los años 2008-2020

\begin{tabular}{|c|c|c|c|c|c|c|c|}
\hline \multirow[t]{2}{*}{ Año } & \multicolumn{2}{|c|}{ Promedio CTG } & \multicolumn{2}{|c|}{$\begin{array}{c}\text { Promedio global curso } \\
\text { teórico }\end{array}$} & \multirow[t]{2}{*}{$\mathbf{n}$} & \multirow{2}{*}{$\begin{array}{c}\text { Media de } \\
\text { diferencias } \\
\text { relacionadas }\end{array}$} & \multirow[t]{2}{*}{ Valor $p$} \\
\hline & Media & DS & Media & DS & & & \\
\hline 2008 & 6,1 & 0,52 & 6,1 & 0,41 & 106 & 0,04 & $<0,05$ \\
\hline 2009 & 5,9 & 0,45 & 6,1 & 0,34 & 101 & $-0,17$ & $<0,05$ \\
\hline 2010 & 6 & 0,54 & 5,8 & 0,45 & 112 & 0,19 & $<0,05$ \\
\hline 2011 & 6,6 & 0,22 & 6,1 & 0,39 & 117 & 0,54 & $<0,05$ \\
\hline 2012 & 6,3 & 0,36 & 6 & 0,36 & 115 & 0,28 & $<0.05$ \\
\hline 2013 & 6,4 & 0,34 & 6 & 0,39 & 105 & 0,39 & $<0.05$ \\
\hline 2014 & 6,4 & 0,44 & - & - & 124 & - & - \\
\hline 2015 & 6,3 & 0,39 & 5,9 & 0,44 & 122 & 0,34 & $<0.05$ \\
\hline 2016 & 6,1 & 0,43 & 6,1 & 0,42 & 120 & $-0,02$ & 0,58 \\
\hline $2017-1 *$ & 6 & 0,59 & - & - & 110 & - & - \\
\hline $2017-2^{*}$ & 6 & 0,6 & 6,3 & 0,29 & 96 & $-0,33$ & $<0,05$ \\
\hline 2018 & 5,8 & 0,59 & 6,0 & 0,40 & 116 & $-0,19$ & $<0,05$ \\
\hline $2020 * *$ & 6,3 & 0,52 & - & - & 122 & - & - \\
\hline
\end{tabular}

*En 2017 dos generaciones de estudiantes de la EMPUC cursaron el CTG. 2017-1 corresponde al último capítulo dictado en contexto del diseño curricular tradicional (dentro del curso Integrado de Clínica III y IV), mientras que 2017-2 corresponde la primera versión realizada en el currículo reformado (dentro del curso Clínicas II y III). **El CTG no fue impartido el año 2019 , sino que se dictó durante el primer semestre de 2020.

ítem "Fuentes de información" (Tabla 5). Existieron diferencias significativamente estadísticas en todas las áreas evaluadas $(\mathrm{p}<0,05)$.

\section{Calificaciones finales de los alumnos}

El sistema de calificaciones se basa en una escala del 1 al 7, con un porcentaje de exigencia establecido en $60 \%$ para la nota mínima aprobatoria (correspondiente a un 4,0). Como se puede observar en la Tabla 6, históricamente el CTG tiende a mantener un promedio final igual o significativamente mayor al del curso teórico en que se enmarca. Sin embargo, no fue factible acceder a la totalidad de datos necesaria para evaluar con certeza el comportamiento en el tiempo de las calificaciones finales de los alumnos, ni su posible correlación con los cambios curriculares implementados.

\section{Discusión}

El CTG constituye la principal instancia teórica del currículo de pregrado para aprender gastroen- 
terología en la EMPUC. A lo largo de los años se han realizado múltiples modificaciones acordes a los cambios de malla curricular, incorporación de nuevas herramientas en educación médica y el uso apropiado de las tecnologías 9 . Por ejemplo, la incorporación de nuevas herramientas basadas en e-learning ha demostrado aumentar la percepción de aprendizaje de los alumnos y optimizar los tiempos de enseñanza, sin afectar el aprendizaje de los alumnos en estudios previos ${ }^{10}$.

Entre los cambios realizados al programa del CTG, se evidenció una reestructuración de las instancias integrativas a través de una metodología centrada en el aprendizaje activo. Un ejemplo lo constituye la incorporación de sesiones interactivas en base a casos clínicos, que permiten poner en práctica los conocimientos adquiridos y resolver dudas del capítulo.

La metodología de clase invertida fomenta el autoaprendizaje activo, permitiendo dedicar las sesiones presenciales a actividades de aprendizaje centradas en el estudiante ${ }^{11-13}$. La incorporación de clases videograbadas constituye un hito innovador, que facilita el acceso a las clases y busca servir de material de apoyo en el proceso de aprendizaje. Sin embargo, resultaría necesario realizar un estudio dirigido para probar su impacto en la satisfacción y el rendimiento del alumnado. En este sentido, se ha planificado transformar de forma progresiva las clases tradicionales a formato de clase invertida.

Por su parte, las jornadas de investigación en Gastroenterología buscan motivar al alumnado en aspectos relacionados con la generación de conocimiento y aproximarlo de forma precoz a la investigación. Este espacio ha impactado en la publicación colaborativa de diversos artículos de Medicina Basada en la Evidencia $(n=26$ desde 2015), y ha acercado al estudiantado a los proyectos de investigación del Departamento de Gastroenterología de la institución.

El proceso de evaluación para el aprendizaje, diseñado bajo el paradigma de que el sistema de evaluación está intrínsecamente ligado al proceso educacional ${ }^{9}$, ha demostrado tener un impacto positivo en la percepción de los alumnos a través de la encuesta del capítulo en las áreas "Notas" y "Feedback". Esto podría deberse al efecto positivo que tiene la evaluación sumativa en la organización del estudio del alumnado, mediante una distribución más equitativa de los contenidos de- mandados a través del tiempo. En segundo lugar, la mejoría en el segundo ítem mencionado puede atribuirse al desarrollo de instancias obligatorias de Feedback, donde los alumnos son invitados a exponer sus dudas y comentarios respecto tanto al ámbito académico como organizacional del CTG.

Resulta interesante destacar que el CTG es el único capítulo del curso teórico que cuenta con un manual propio, lo que ha sido valorado positivamente por el alumnado. Este material es accesible a todos los estudiantes, favoreciendo la preparación autogestionada previa a las clases expositivas o videograbadas.

La incorporación de una nueva plataforma de gestión del aprendizaje en EMPUC, permitió, en contexto de la pandemia por COVID-19 en 2020, transitar rápidamente desde un curso con componentes semipresenciales a una modalidad completamente a distancia (incluyendo nuevas clases videograbadas, videoconferencias, videoseminarios y evaluaciones en línea), posibilitando mantener el calendario original del curso y la interacción docente-alumno en tiempo real, con excelentes resultados académicos y una muy buena percepción por parte del estudiantado.

Las medidas tomadas han recibido una valoración subjetiva positiva, probablemente condicionada por un nuevo enfoque de enseñanza, debidamente fundado y orientado a un contexto generacional en específico. Si bien el capítulo mantiene de forma consistente calificaciones no inferiores a las del curso teórico que lo contiene, son necesarios nuevos instrumentos para conocer en detalle el impacto de las medidas adoptadas sobre la aprehensión de contenidos por parte del estudiantado. En la Tabla 7 se describen oportunidades de mejora en el CTG.

Entender la enseñanza como un proceso en constante desarrollo y transformación, nos permite asumir el desafío de una entrega de conocimiento médico ajustada a las necesidades, inquietudes y características de su audiencia, siendo deber de toda institución educacional adecuar sus modificaciones curriculares a los cambios personales y generacionales del estudiantado.

En conclusión, el CTG se ha sometido a una serie de modificaciones curriculares orientadas a entregar contenidos gastroenterológicos básicos de forma amigable, intuitiva y práctica, apoyándose en un modelo de aprendizaje activo, clases invertidas y evaluación dirigida para el apren- 
Tabla 7. Oportunidades de mejora identificadas en el Curso Teórico de Gastroenterología y posibles indicadores para su evaluación

\begin{tabular}{|c|c|c|}
\hline $\begin{array}{l}\text { Oportunidad/Material } \\
\text { existente }\end{array}$ & Oportunidad de mejora & Indicador \\
\hline Jornadas de investigación & $\begin{array}{l}\text { Presentación de pósters electrónicos por parte de } \\
\text { los estudiantes }\end{array}$ & $\begin{array}{l}\text { Percepción, habilidades de investigación, } \\
\text { rendimiento }\end{array}$ \\
\hline Autoinstructivos y manual & $\begin{array}{l}\text { Clase invertida } \text { Canvas }^{\oplus} \text { : Análisis de material y } \\
\text { desarrollo de actividades en formato no presencial } \\
\text { para luego aplicarlo en discusión de un caso } \\
\text { clínico en clase }\end{array}$ & $\begin{array}{l}\text { Percepción, rendimiento, cambios en } \\
\text { habilidades de resolución de casos clíni- } \\
\text { cos y optimización del tiempo presencial }\end{array}$ \\
\hline $\begin{array}{l}\text { Clases videograbadas: } \\
\text { teórico }\end{array}$ & $\begin{array}{l}\text { Clase invertida } \text { Canvas }^{\odot} \text { : Revisión de video o au- } \\
\text { dioclase con cuestionarios que tienen que resolver } \\
\text { en formato no presencial para luego aplicarlo en } \\
\text { discusión de un caso clínico en clase }\end{array}$ & $\begin{array}{l}\text { Percepción y rendimiento en cuestiona- } \\
\text { rios y cambios en habilidades de resolu- } \\
\text { ción de casos clínicos y optimización del } \\
\text { tiempo presencial }\end{array}$ \\
\hline $\begin{array}{l}\text { Clases videograbadas: } \\
\text { procedimental }\end{array}$ & $\begin{array}{l}\text { Entrenamiento no presencial de procedimientos: } \\
\text { Los estudiantes pueden revisar las clases video- } \\
\text { grabadas de procedimientos y luego subir sus } \\
\text { propios videos realizando el procedimiento a } \\
\text { Canvas }^{\odot} \text {. La plataforma permite que estos videos } \\
\text { en formato tarea puedan ser corregidos por pares } \\
\text { y por un profesor con una pauta de observación } \\
\text { o rúbrica }\end{array}$ & $\begin{array}{l}\text { Percepción, rendimiento en procedi- } \\
\text { mientos, feedback de pares, variabilidad } \\
\text { inter-evaluador entre pares y profesor }\end{array}$ \\
\hline Sesiones grupos pequeños & $\begin{array}{l}\text { Trabajo con Kahoot o Mentimeter, realizar clases } \\
\text { con participación de audiencia en base a algún } \\
\text { caso clínico representado en video. Con preguntas } \\
\text { a través de estos programas, los estudiantes pue- } \\
\text { den mejorar su razonamiento clínico participando } \\
\text { en la toma de decisiones resolución de problemas } \\
\text { de un paciente o caso clínico virtual }\end{array}$ & $\begin{array}{l}\text { Percepción, cambios en la participación } \\
\text { de la clase, rendimiento en la resolución } \\
\text { del caso }\end{array}$ \\
\hline
\end{tabular}

dizaje. Estos cambios se han reflejado en una mayor percepción de feedback y disponibilidad de material de estudio, sin deterioro en el desempeño académico de los estudiantes.

Agradecimientos: A la Dirección de Pregrado y al Centro de Educación Médica de la Escuela de Medicina de la Pontificia Universidad Católica, en especial a Karina Soto y Dr. Bruno Grassi por facilitar el acceso a la información necesaria para la publicación.

\section{Referencias}

1. Cisternas M, Rivera S, Sirhan M, Thöne N, Valdés C, Pertuzé J, et al. Reforma curricular de la carrera de Medicina de la Pontificia Universidad Católica de Chile. Rev Med Chile 2016; 144 (1): 102-7.
2. Bitran M, Zúñiga D, Pedrals N, Padilla O, Mena B. Medical students' change in learning styles during the course of the undergraduate program: from 'thinking and watching' to 'thinking and doing'. Canadian Medical Education Journal 2012, 3(2).

3. Bitran M, Zúñiga D, Leiva I, Calderón M, Tomicic A, Padilla O, Riquelme A. ¿Cómo aprenden los estudiantes de medicina en la transición hacia el ciclo clínico? Estudio cualitativo de las percepciones de estudiantes y docentes acerca del aprendizaje inicial de la clínica? Rev Med Chile 2014; 142: 723-31.

4. Waljee JF, Chopra V, Saint S. Mentoring millennials. JAMA 2018; 319 (15): 1547-8.

5. Kattan E, Pérez G, Le Roy C, Sirhan M, González A, Rybertt T, et al. Proceso de mejoría de pruebas de conocimiento con preguntas de selección múltiple en un curso teórico de Pregrado de Medicina. Rev Educ Cienc Salud 2014; 11 (2): 116-23.

6. Buckley S, Coleman J, Davison I, Khan KS, Zamora J, 
Malick S, et al. The educational effects of portfolios on undergraduate student learning: A Best Evidence Medical Education (BEME) systematic review. BEME Guide No. 11. Med Teach 2009; 31 (4): 282-98.

7. Riquelme A, Méndez B, De La Fuente P, Padilla O, Benaglio C, Sirhan M, et al. Desarrollo y validación de encuesta de percepción del portafolio en estudiantes de medicina de pregrado. Rev Med Chile 2011; 139 (1): 45-53.

8. Pérez G, Kattan E, Collins L, Wright AC, Rybertt T, González A, et al. Evaluación para el aprendizaje: experiencia en un curso teórico de pregrado en medicina. Rev Med Chile 2015; 143 (3): 329-36.

9. Mehta NB, Hull AL, Young JB, Stoller JK. Just imagine: new paradigms for medical education. Acad Med 2013; 88 (10): 1418-23.

10. Carbonaro M, King S, Taylor E, Satzinger F, Snart F, Drummond J. Integration of e-learning technologies in an interprofessional health science course. Med Teach 2008; 30 (1): 25-33.

11. Granero Lucchetti AL, Ezequiel ODS, Oliveira IN, Moreira-Almeida A, Lucchetti G. Using traditional or flipped classrooms to teach "Geriatrics and Gerontology"? Investigating the impact of active learning on medical students' competences. Med Teach 2018; 40 (12): 1248-56.

12. Lockman K, Haines ST, McPherson ML. Improved Learning Outcomes After Flipping a Therapeutics Module: Results of a Controlled Trial. Acad Med 2017; 92 (12): 1786-93.

13. McLaughlin JE, Roth MT, Glatt DM, Gharkholonarehe N, Davidson CA, Griffin LM, et al. The flipped classroom: a course redesign to foster learning and engagement in a health professions school. Acad Med 2014; 89 (2): 236-43. 